\title{
Survival and metabolic activity of selected strains of Propionibacterium freudenreichii in the gastrointestinal tract of human microbiota-associated rats
}

\author{
Annaig Lan ${ }^{1,2}$, Aurélia Bruneau ${ }^{3}$, Catherine Philippe ${ }^{3}$, Violaine Rochet ${ }^{3}$, Annette Rouault ${ }^{1}$, \\ Christophe Hervé ${ }^{2}$, Nathalie Roland ${ }^{2}$, Sylvie Rabot ${ }^{3}$ and Gwénaël $\mathrm{Jan}^{1 *}$ \\ ${ }^{1}$ INRA, Agrocampus Rennes, UMR1253, Science et Technologie du Lait et de I'Oeuf, F-35000 Rennes, France \\ ${ }^{2}$ Laboratoires Standa, 68 rue R. Kaskoreff, 14050 Caen Cedex 4, France \\ ${ }^{3}$ INRA, Unité d'Ecologie et Physiologie du Système Digestif, 78352 Jouy-en-Josas Cedex, France
}

(Received 22 August 2006 - Revised 23 October 2006 - Accepted 26 October 2006)

\begin{abstract}
In addition to their use in cheese technology, dairy propionibacteria have been identified as potential probiotics. However, to have a probiotic effect, propionibacteria have to survive and to remain metabolically active in the digestive tract. The aim of the present study was to investigate the survival and metabolic activity of Propionibacterium freudenreichii within the gastrointestinal tract of human microbiota-associated rats, and its influence on intestinal microbiota composition and metabolism. Twenty-five dairy Propionibacterium strains were screened for their tolerance towards digestive stresses and their ability to produce propionate in a medium mimicking the content of the human colon. Three strains were selected and a daily dose of $2 \times 10^{10}$ colony-forming units was fed to groups of human microbiota-associated rats for $20 \mathrm{~d}$ before microbiological, biochemical and molecular investigations being carried out. These strains all reached 8-log values per g faeces, showing their ability to survive in the gastrointestinal tract. Transcriptional activity within the intestine was demonstrated by the presence of $P$. freudenreichii-specific transcarboxylase mRNA. The probiotic efficacy of propionibacteria was yet species- and strain-dependent. Indeed, two of the strains, namely TL133 and TL1348, altered the faecal microbiota composition, TL133 also increasing the caecal concentration of acetate, propionate and butyrate, while the third strain, TL3, did not have similar effects. Such alterations may have an impact on gut health and will thus be taken into consideration for further in vivo investigations on probiotic potentialities of $P$. freudenreichii.
\end{abstract}

Probiotics: Propionibacteria: Short-chain fatty acids: Intestinal microbiota

A probiotic is generally defined as 'a live micro-organism which, when administered in adequate amounts, confers a health benefit on the host' (Food and Agriculture Organization \& World Health Organization, 2002). The main claimed effects for probiotics are improvement of lactose digestion, prevention of intestinal disturbances, treatment and prevention of antibiotic or acute diarrhoea, reduction of intestinal infection and colonisation by pathogenic bacteria, alleviation of irritable bowel syndrome and of inflammatory bowel disease and modulation of colon carcinogenesis (Marteau et al. 2001). The reported mechanisms that may explain the healthpromoting effects of probiotics include improvement of the gut mucosal barrier, immunomodulation (immune response balance), modulation of the intestinal microbiota (allowing an equilibrium between the populations of beneficial and potentially harmful bacteria) and of the corresponding metabolic activities within the gut, leading to decreased toxigenic reactions, enhanced production of SCFA and vitamins, bile salt deconjugation or lactose hydrolysis (Ouwehand et al. 2002; Saarela et al. 2002). The criteria for probiotic selection include safety (strain, species and genus safety properties and non-pathogenicity), technological properties (tolerance towards probiotic product processing and storage), survival in the digestive tract, which is linked with stress adaptation, and at least one property beneficial to human health. Moreover, human origin and adhesion to human epithelial cells may also be considered as selection criteria for probiotics. Most of the probiotics used are lactobacilli or bifidobacteria, but some probiotic effects have also been reported for some Bacillus strains, a non-pathogenic strain of Escherichia coli, some yeasts and propionibacteria, mainly Propionibacterium freudenreichii (Ouwehand et al. 2002).

Like lactobacilli, dairy propionibacteria have a long history of safe use as starter cultures in the food industry. For the manufacture of Swiss-type cheeses, $P$. freudenreichii has received 'generally recognised as safe' (GRAS) status by the US Food and Drug Administration (21CFR133.195). Besides this application, they are also studied for their probiotic abilities. In man, dairy propionibacteria survive, at least partially, within the digestive tract (Bougle et al. 1999; Jan et al. 2002b) and selected strains

\footnotetext{
Abbreviations: CFU, colony-forming unit; Cy5, indodicarbocyanine; FISH, fluorescent in situ hybridisation; Tris, tri(hydroxymethyl)-aminomethane. * Corresponding author: Dr Gwénaël Jan, fax +332234853 50, email gjan@rennes.inra.fr
} 
stimulate the growth of bifidobacteria (Bougle et al. 1999; Satomi et al. 1999; Hojo et al. 2002). In rodents, different propionibacteria have been shown to have immunomodulatory effects (Perez Chaia et al. 1995; Kirjavainen et al. 1999). Propionibacteria also decreased activity of intestinal enzyme involved in the conversion of carcinogenic compounds, for example, $\beta$-glucuronidase (Perez Chaia et al. 1999), adhered to intestinal epithelium of mice (Zarate et al. 2002) and reduced the severity of chemically induced colitis in rats (Michel et al. 2005). Furthermore, propionibacteria are able to inhibit the growth of pathogenic micro-organisms (Lyon et al. 1993) and to produce bacteriocins and vitamin $\mathrm{B}_{12}$ (Holo et al. 2002; Hugenholtz et al. 2002).

Moreover, propionibacteria harbour a particular central carbon metabolic pathway, the propionic fermentation, which involves the Wood-Werkman cycle (Wood, 1981) and requires a multimeric transcarboxylase (methylmalonylCoA carboxyltransferase; EC 21.3.1). This peculiar metabolism allows conversion of various substrates into $\mathrm{CO}_{2}$ and the SCFA acetate and propionate. SCFA, particularly butyrate, are known to have an important influence on colonic health (Mortensen \& Clausen, 1996). This characteristic may constitute a key probiotic potential for propionibacteria, as it has been previously shown that the SCFA butyrate is a potent inducer of apoptosis in colon cancer cells in vitro (Scheppach et al. 1995). Regarding the major endproducts of dairy propionibacteria, acetate and propionate were shown to kill human adenocarcinoma cell lines by apoptosis during co-cultures with the dairy species $P$. freudenreichii and $P$. acidipropionici (Jan et al. 2002a), a property that could help colon cancer prevention or treatment.

However, to have such health effects, the potential probiotic must tolerate digestives stresses and reach significant populations within the gut. Stress tolerance is highly strain-dependent within the $P$. freudenreichii species (Jan et al. 2000; Anastasiou et al. 2006). Occurrence of propionic fermentation within the colon content also remains a key question for dairy propionibacteria probiotic application. The aim of the present study was first to screen in vitro a set of dairy propionibacteria, known for their technological properties, with respect to their tolerance towards digestive stresses and their production of propionate in a medium mimicking the content of the human colon. Second, after in vitro selection, the best candidates were administered to human microbiota-associated rats in order to evaluate propionibacteria survival and metabolic activity within the gastrointestinal tract, and to study the influence of $P$. freudenreichii on intestinal metabolism and human microbiota composition.

\section{Material and methods}

\section{Bacterial strains and growth conditions}

The Propionibacterium strains (Table 1) originated from our local TL propionibacteria strain collection (INRA, Agrocampus Rennes, UMR STLO). They were kept frozen $\left(-80^{\circ} \mathrm{C}\right)$ as glycerol stocks using our standard procedure and routinely cultivated on a modified yeast extract-lactate medium containing $144 \mathrm{~mm}$-sodium lactate (Malik et al. 1968). Growth was carried out at $30^{\circ} \mathrm{C}$ without shaking and monitored spectrophotometrically at $650 \mathrm{~nm}$ as well as by colony-forming unit (CFU) counting.

\section{In vitro assays}

Digestive stress challenge. After CFU counting, early stationary-phase cultures $(3 \mathrm{~d})$ were diluted 10 -fold in a

Table 1. Dairy Propionibacterium strains used in the present study

\begin{tabular}{llll}
\hline Strain & \multicolumn{1}{c}{ Species } & Subspecies & Origination \\
\hline TL125 & Propionibacterium freudenreichii & shermanii & Dairy product \\
TL133 & P. freudenreichii & shermanii & Dairy product \\
TL134 & P. freudenreichii & shermanii & Dairy product \\
TL138 & P. freudenreichii & shermanii & Dairy product \\
TL142 & P. freudenreichii & freudenreichii & Dairy product \\
TL144 & P. freudenreichii & shermanii & Dairy product \\
TL160 & P. freudenreichii & shermanii & Dairy product \\
TL165 & P. freudenreichii & shermanii & Dairy product \\
TL166 & P. freudenreichii & shermanii & Dairy product \\
TL162 & P. freudenreichii & shermanii & Dairy product \\
TL1343 & P. freudenreichii & freudenreichii & Human intestine* \\
TL1348 & P. freudenreichii & shermanii & Human intestine* \\
TL1351 & P. freudenreichii & shermanii & Human intestine* \\
TL1360 & P. freudenreichii & shermanii & Human intestine \\
TL33 & P. freudenreichii & freudenreichii & Dairy product \\
TL34 & P. freudenreichii & shermanii & Dairy product \\
TL2 & P. acidipropionici & - & Dairy product \\
TL3 & P. freudenreichii & freudenreichii & Dairy product \\
TL19 & P. freudenreichii & freudenreichii & Dairy product \\
TL63 & P. freudenreichii & shermanii & Dairy product \\
TL213 & P. freudenreichii & shermanii & Dairy product \\
TL502 & P. freudenreichii & freudenreichii & Dairy product \\
TL505 & P. freudenreichii & shermanii & Dairy product \\
TL635 & P. freudenreichii & shermanii & Dairy product \\
TL1123 & P. freudenreichii & shermanii & Dairy product \\
\hline *Strains isolated from human faecal samples after a 4-week wash-out period without dairy propio- \\
nibacteria ingestion. & & \\
& & &
\end{tabular}


pre-warmed $\left(37^{\circ} \mathrm{C}\right)$ acidified ( $\mathrm{pH}$ adjusted to 3.3 using $\mathrm{HCl}$ ) lactate broth (Jan et al. 2000); the mixture led to a final $\mathrm{pH}$ of 3.5. Propionibacteria were acid-challenged at $37^{\circ} \mathrm{C}$ for $1 \mathrm{~h}$ before centrifugation $(10 \mathrm{~min} ; 10000 \mathrm{~g})$ and re-suspended in pre-warmed $\left(37^{\circ} \mathrm{C}\right)$ peptone water $(0.1 \%$ peptic digest of meat; Biokar Diagnostics, Beauvais, France), $\mathrm{pH} 7 \cdot 0$, containing $0.9 \% \mathrm{NaCl}$, in order to stop acid stress. These cell suspensions were then diluted 5-fold in a pre-warmed yeast extract-lactate medium containing $0.1 \%(\mathrm{w} / \mathrm{v})$ dried ox bile (Biokar Diagnostics) and challenged at $37^{\circ} \mathrm{C}$ for $2 \mathrm{~h}$. Bile challenge was stopped by dilution and surviving cells were enumerated on solidified yeast extract-lactate medium after $7 \mathrm{~d}$ of anaerobic incubation at $30^{\circ} \mathrm{C}$ for maximal recovery of living bacteria as described previously (Leverrier et al. 2003).

Short-chain fatty acid production. Early stationary-phase propionibacteria yeast extract-lactate cultures were used to inoculate $(1 \%)$ a pre-warmed medium, designed to mimic the content of the human colon (Gibson \& Wang, 1994), and modified by the addition of sodium lactate to facilitate propionic fermentation (Table 2). Following incubation $\left(37^{\circ} \mathrm{C} ; 24 \mathrm{~h}\right)$, cultures were centrifuged $(15 \mathrm{~min} ; 10000 \mathrm{~g})$ and supernatant fractions diluted in $0.005 \mathrm{M}-\mathrm{H}_{2} \mathrm{SO}_{4}$ before filtration $(0.2 \mu \mathrm{m})$ and chromatographic analysis. SCFA were analysed by HPLC (Gold; Beckman Coulter Corp., Fullerton, CA, USA) using UV detection at $210 \mathrm{~nm}$. The anion exchange column $\left(300 \times 6 \mathrm{~mm}\right.$, Aminex $\mathrm{A}_{6}$; Bio-Rad, Hercules, CA, USA) was operated at room temperature with 0.005

Table 2. Composition of the medium used for short-chain fatty acid production screening

\begin{tabular}{|c|c|}
\hline Constituent & Concentration $(\mathrm{g} / \mathrm{l})$ \\
\hline Pectin & 0.5 \\
\hline Xylan & 0.5 \\
\hline Mucin & 0.5 \\
\hline Starch & 0.5 \\
\hline Peptone & 0.5 \\
\hline Tryptone & 2.5 \\
\hline Yeast extract & 0.5 \\
\hline Bile salts & 0.05 \\
\hline $\mathrm{K}_{2} \mathrm{HPO}_{4}$ & 2 \\
\hline $\mathrm{NaHCO}_{3}$ & 0.2 \\
\hline $\mathrm{NaCl}$ & 4.5 \\
\hline $\mathrm{MgSO}_{4} \cdot 7 \mathrm{H}_{2} \mathrm{O}$ & 0.5 \\
\hline $\mathrm{CaCl}_{2} \cdot 2 \mathrm{H}_{2} \mathrm{O}$ & 0.45 \\
\hline $\mathrm{MnCl}_{2} \cdot 2 \mathrm{H}_{2} \mathrm{O}$ & 0.2 \\
\hline Haemin & 0.05 \\
\hline $\mathrm{FeSO}_{4} .7 \mathrm{H}_{2} \mathrm{O}(\mathrm{mg} / \mathrm{l})$ & 5 \\
\hline $\mathrm{CoCl}_{2} \cdot 6 \mathrm{H}_{2} \mathrm{O}$ & 0.05 \\
\hline Tween $80(\mathrm{ml} / \mathrm{l})$ & 2 \\
\hline Sodium lactate $(60 \%)$ & 5 \\
\hline Thiamin $\mathrm{HCl}(\mu \mathrm{g} / \mathrm{l})$ & 4 \\
\hline Calcium pantothenate $(\mu \mathrm{g} / \mathrm{l})$ & 10 \\
\hline Nicotinic acid $(\mu \mathrm{g} / \mathrm{l})$ & 5 \\
\hline 4-Aminobenzoic acid $(\mu \mathrm{g} / \mathrm{l})$ & 5 \\
\hline Biotin $(\mu \mathrm{g} / \mathrm{l})$ & 2 \\
\hline Vitamin $\mathrm{B}_{12}(\mu \mathrm{g} / \mathrm{l})$ & 0.5 \\
\hline Cysteine & 0.8 \\
\hline $\mathrm{PH}$ & 6.0 \\
\hline Distilled water & Up to 1 litre \\
\hline
\end{tabular}

$\mathrm{M}-\mathrm{H}_{2} \mathrm{SO}_{4}(0.5 \mathrm{ml} / \mathrm{min})$ as eluent. Standard solutions of lactic acid, acetic acid and propionic acid of known concentrations were used for column calibration.

\section{Animals}

Twenty-four adult male Fischer 344 rats were used. They were born germ-free and bred in germ-free conditions in the GermFree Rodent Breeding Facilities of Unité d'Ecologie et Physiologie du Système Digestif (INRA, Jouy-en-Josas, France), according to established methods (Coates, 1968). Rats were aged 12 weeks at the start of the experiment (mean weight 291 (SEM 4)g). They were randomly separated into four groups of six animals housed in four sterile Plexiglas isolators (Ingénia, Vitry-sur-Seine, France). Within each isolator, rats were kept in pairs in standard macrolon cages containing a bed of wood shavings. They were given free access to autoclaved tap water and a pelleted semi-synthetic diet (Scientific Animal Food and Engineering, Augy, France) sterilised by $\gamma$ irradiation at $45 \mathrm{kGy}$ (IBA Mediris, Fleurus, Belgium). To reproduce the diversity of a human-type diet, the food contained lipids and proteins of animal and plant origins, sucrose and cooked starch (Table 3). Analytical compounds of DM were: crude proteins, $18 \%$; crude fat, $8 \%$; ash, 6\%; carbohydrates $68 \%$ (energy $19.33 \mathrm{MJ} / \mathrm{kg} \mathrm{DM}$; Eurofins Scientific Analytics, Nantes, France). Throughout the study, isolators were maintained in controlled conditions of light (07.00-19.00 hours), temperature $\left(20-22^{\circ} \mathrm{C}\right)$ and humidity $(45-55 \%)$.

\section{Experimental design}

All procedures were carried out in accordance with the European guidelines for the care and use of laboratory animals. On day 1 , all rats were orally administered with $1 \mathrm{ml}$ of a whole fresh faecal suspension made from the stools of a healthy

Table 3. Composition of the diet

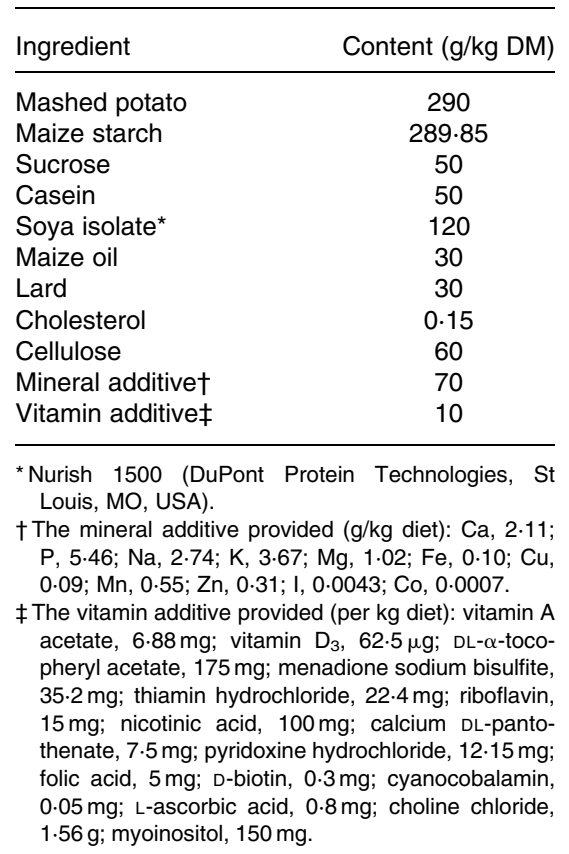


adult human subject. This subject had followed a normal diet over 3 weeks but with the exclusion of fermented products containing propionibacteria (no consumption of Swiss-type and other pressed cheeses) in order to avoid the presence of propionibacteria in faecal content. Fresh stools $(2 \mathrm{~g})$ were transferred in an anaerobic glove box and dispersed in $200 \mathrm{ml}$ Brain Heart Infusion broth (Difco, Becton Dickinson, Le Pont de Claix, France); the suspension was subsequently transferred in the isolators and given to rats using a sterile stainless-steel stomach tube. The rats were given 3 weeks to allow the microbiota to settle in the digestive tract and the rat physiology to adapt to the new bacterial status. On day 21 , the human microbiota-associated rats were randomly allocated to four treatments, namely control, TL3, TL133 and TL1348 strains. Each rat was orally administered daily for 3 weeks either with $1.0 \mathrm{ml}$ of physiological saline solution (control group) or with $2 \times 10^{10} \mathrm{CFU}$ of the corresponding $P$. freudenreichii strain (TL3, TL133 or TL1348) suspended in $1.0 \mathrm{ml}$ of physiological saline solution. The bacterial suspensions were prepared every day from early stationaryphase propionibacteria cultures.

On day 21 (initial time), day 31 (medium time) and day 41 (final time), faecal pellets were freshly collected from each rat and divided into two samples. One was stored at $-80^{\circ} \mathrm{C}$ until analysis of bacterial enzymic activities. The second was immediately 10-fold diluted with a dilution medium (casein enzymic hydrolysate $(2 \mathrm{~g} / \mathrm{l})$, yeast extract $(2 \mathrm{~g} / \mathrm{l})$, $\left.\mathrm{NaCl}(5 \mathrm{~g} / \mathrm{l}), \mathrm{KH}_{2} \mathrm{PO}_{4}(1 \mathrm{~g} / \mathrm{l}) ; \mathrm{pH} 7.0\right)$. Of this dilution, $1 \mathrm{ml}$ was used for enumeration of propionibacteria and one volume of this dilution was added to three volumes of $4 \%$ paraformaldehyde for overnight fixation, then stored at $-80^{\circ} \mathrm{C}$ until analysis of the microbiota by fluorescent in situ hybridisation (FISH) (Rigottier-Gois et al. 2003a).

On day 42, rats were weighed and killed by $\mathrm{CO}_{2}$ asphyxiation. The caecum was removed, the caecal $\mathrm{pH}$ was measured and the content was weighed. Two samples were used immediately for enumeration of propionibacteria and for RNA extraction followed by RT-PCR. The remaining part was distributed into several vials stored at $-80^{\circ} \mathrm{C}$ for SCFA analysis.

\section{Enumeration of propionibacteria in faecal and caecal samples}

Freshly collected faeces and caecal contents were immediately dispersed in the dilution medium. Samples from serial 10-fold dilutions were poured into the Pal-Propiobac ${ }^{\circledR}$ selective agar (Laboratoires Standa, Caen, France) added with metronidazole $(4 \mathrm{mg} / \mathrm{l})$ for propionibacteria enumeration in samples of intestinal origin, as described previously (Jan et al. 2002b). Plates were then incubated anaerobically at $30^{\circ} \mathrm{C}$ for 1 week before colony counting. Results were expressed as $\log \mathrm{CFU} / \mathrm{g}$ faeces or caecal content.

\section{Analysis of bacterial enzymic activities in faecal samples}

$\beta$-Glucuronidase and $\beta$-galactosidase activities were measured spectrophotometrically $(\lambda 400 \mathrm{~nm})$ by the rate of release of $p$-nitrophenol from the $p$-nitrophenylglycoside as described by Andrieux et al. (1998). Analyses were performed in duplicate. Enzymic activity was expressed as $\mu \mathrm{mol}$ product formed/ min per $g$ wet faeces.
Ribonucleic acid extraction from caecal samples and cDNA synthesis

In order to detect $P$. freudenreichii transcarboxylase $5 \mathrm{~S}$ mRNA (Hervé et al. 2006), total caecal microbial RNA were isolated using the Nucleospin RNA II kit (Macherey Nagel, Düren, Germany) according to the manufacturer's instructions but with modifications and partially adapted steps as described below. Into a $2 \mathrm{ml}$ microtube (VWR, Nogent sur Marne, France), $60 \mathrm{mg}$ of caecal sample were mixed with 300 to $500 \mathrm{mg}$ of $\mathrm{Zr}$ beads (100 $\mu \mathrm{m}$; VWR), $100 \mu \mathrm{l}$ of cold TE 1x, $350 \mu \mathrm{l}$ of buffer RA1 (supplied with the kit) and $3.5 \mu \mathrm{l}$ of $\beta$-mercaptoethanol. After homogenisation with vortex at maximal speed, the tubes containing the $\mathrm{Zr}$ beads were shaken twice at $2000 \mathrm{~g}$ for $40 \mathrm{~s}$ in a Fast Prep instrument (FP 120, Bio101; Savant, Holbrook, NY, USA) with rapid cooling on ice between steps. After shaking, the tubes were centrifuged at $7000 \mathrm{~g}$ for $2 \mathrm{~min}$. The supernatant fractions were transferred to new tubes, and total RNA were rapidly subjected to chloroform-phenol extraction and ethanol precipitation. Pellets were washed with $70 \%$ ethanol and re-suspended in $120 \mu \mathrm{l}$ of RNAse-free water. To remove contaminating DNA, a DNAse treatment was performed on $20 \mu \mathrm{g}$ of total nucleic acid with deoxyribonuclease I, amplification grade (Invitrogen, Cergy Pontoise, France). Following incubation at $30^{\circ} \mathrm{C}$ for $30 \mathrm{~min}$, RNA were concentrated to $20 \mu \mathrm{l}$ with the RNeasy ${ }^{\circledR}$ MinElute Cleanup (Qiagen, Hilden, Germany). RNA integrity was determined after electrophoresis on agarose gel containing ethidium bromide. RNA concentration and purity were estimated by the Ribogreen ${ }^{\circledR}$ RNA Quantification Kit (Molecular Probes, Eugene, OR, USA) and by spectrofluorometry (Kontron Instruments, Rungis, France), respectively. One portion of $10 \mu$ l (approximately $10 \mu \mathrm{g}$ ) of concentrated RNA was reverse-transcribed to cDNA using Superscript II RT (Invitrogen, Cergy-Pontoise, France) with 100 pmol of the reverse primer (Hervé et al. 2006). In parallel, the remaining $10 \mu \mathrm{l}(10 \mu \mathrm{g})$ of RNA were treated in the same way, except for the absence of RT, and were considered as a negative control. After the reaction, the synthesised cDNA was purified using Qiaquick ${ }^{\circledR}$ PCR Purification kit (Qiagen).

The reaction mixture of the TaqMan ${ }^{\mathrm{TM}}$ real-time PCR amplification (in a final reaction volume of $25 \mu \mathrm{l}$ ) contained $1 \times$ of Quantitect Probe PCR Master Mix (Qiagen), $200 \mathrm{~nm}$ of the probe and $300 \mathrm{~nm}$ of each of the primers. All the primers and probe used in the present study were synthesised by QIAGEN Operon Europe (Cologne, Germany). To detect the transcarboxylase mRNA, $10 \mu \mathrm{l}$ of total cDNA were used. To verify the purity of the amplicon, samples of $10 \mu \mathrm{l}$ were analysed by electrophoresis on a $4 \%(\mathrm{w} / \mathrm{v})$ agarose $3: 1$ (Amresco, Solon, OH, USA) gel containing ethidium bromide.

\section{Short-chain fatty acid analysis in caecal samples}

Samples were water extracted and proteins were precipitated with phosphotungstic acid. A volume of $0.1 \mu \mathrm{l}$ supernatant fraction was analysed for SCFA on a gas-liquid chromatograph (Autosystem XL; Perkin Elmer, Saint-Quentinen-Yvelines, France) equipped with a split-splitless injector, a flame-ionisation detector and a capillary column $(15 \mathrm{~m} \times$ $0.53 \mathrm{~mm}, \quad 0.5 \mu \mathrm{m}$ ) impregnated with SP 1000 (FSCAP 
Nukol; Supelco, Saint-Quentin-Fallavier, France). Carrier gas (He) flow rate was $10 \mathrm{ml} / \mathrm{min}$ and inlet, column and detector temperatures were $175^{\circ} \mathrm{C}, 100^{\circ} \mathrm{C}$ and $280^{\circ} \mathrm{C}$, respectively. 2-Ethylbutyrate was used as the internal standard (Rabot et al. 2000). Samples were analysed in duplicate. Data were collected and peaks integrated using the Turbochrom v. 6 software (Perkin Elmer, Courtaboeuf, France).

\section{Faecal microbiota analysis by fluorescent in situ hybridisation combined with flow cytometry}

FISH analysis was carried out as described previously (Rigottier-Gois et al. 2003a), using the following probes to detect bacterial groups in the faecal samples (Table 4): Eub338 (total bacteria); Ato291 (Atopobium, Eggerthella and Collinsella); Bac303 (Bacteroides and Prevotella); Bif164 (Bifidobacterium); Clep866 (Clostridium leptum); Erec482 (Eubacterium rectale-C. coccoides); Enter1432 (enterobacteria); Lab158 (Lactobacillus and Enterococcus). Oligonucleotidic probes were 5'-labelled with indodicarbocyanine (Cy5) or fluorescein isothiocyanate and purified by HPLC (Integrated DNA Technologies, Coralville, IA, USA). The fixed faecal samples were washed in TE buffer (100 mM-tri(hydroxymethyl)-aminomethane (Tris)$\mathrm{HCl}, 50 \mathrm{~mm}$-EDTA) and permeabilised with lysozyme $(1 \mathrm{mg} / \mathrm{ml})$ in TE buffer for $10 \mathrm{~min}$. After centrifugation, the pellet was washed in PBS and re-suspended in hybridisation buffer ( $0.9 \mathrm{M}-\mathrm{NaCl}, 20 \mathrm{~mm}-$ Tris- $\mathrm{HCl}, \mathrm{pH} 8.0,0.01 \%$ SDS, and $30 \%$ formamide). Of this suspension, $40 \mu \mathrm{l}$ were mixed with $10 \mu \mathrm{l}$ of a mixture containing the fluorescein isothiocyanate-labelled Eub338 probe $(20 \mathrm{ng} / \mu \mathrm{l})$ and one of the group-specific Cy5-labelled probe $(20 \mathrm{ng} / \mu \mathrm{l})$. Hybridisation was performed at $35^{\circ} \mathrm{C}$ in the dark for $16 \mathrm{~h}$; then, a volume of $150 \mu \mathrm{l}$ of hybridisation solution was added to the reaction mixture and cells were pelleted at $4000 \mathrm{~g}$ for $15 \mathrm{~min}$. Non-specific binding of the probes was removed by incubating pelleted cells at $37^{\circ} \mathrm{C}$ for $20 \mathrm{~min}$ in washing buffer $(0.065 \mathrm{M}-\mathrm{NaCl}, 20 \mathrm{~mm}$-Tris- $\mathrm{HCl}, 5 \mathrm{~mm}-$ EDTA, pH 8.0, and 0.01\% SDS). Cells were pelleted again and suspended in $200 \mu \mathrm{l}$ PBS. Samples of $100 \mu \mathrm{l}$ were added with $0.5 \mathrm{ml}$ of FACS Flow (Becton Dickinson, Franklin Lakes, NJ, USA) for data acquisition by flow cytometry, using a FacsCalibur flow cytometer (Becton Dickinson). An air-cooled Ar ion laser $(488 \mathrm{~nm})$ and a red diode laser $(635 \mathrm{~nm})$ were used for excitation, and the green and red signals of the bacteria were collected in the FL1 (515-545 nm) and FL4 (653-669 nm) detectors, respectively. The acquisition threshold was set on side scatter channel and $10^{5}$ fluorescent events were stored in list mode files. Subsequent analysis was performed with the Cell Quest software (Becton Dickinson). An FL1 histogram was built to determine the total number of bacteria present in the sample and hybridising with Eub338. A gate was created in the FL1 histogram and a FL4 histogram was subsequently used to determine the proportion of cells hybridising with the group-specific Cy5 probe. This proportion was corrected by eliminating the background of red fluorescence, determined by using the Non-Eub338Cy5 probe as a negative control. Results were expressed as the proportion of cells hybridising with the group-specific Cy5 probe in relation to the total bacteria hybridising with the Eub338-fluorescein isothiocyanate probe.

\section{Statistical analysis}

The effect of propionibacteria consumption on rats' body and caecal weight, on caecal pH, SCFA concentration and enzymic activities, and on the number of culturable propionibacteria in faeces and caecal contents was analysed using a one-way ANOVA. When ANOVA indicated significant differences, groups were compared in pairs with the StudentNewman-Keuls multiple comparison test. Inter-group similarity of the faecal microbiota at the start of the experiment was checked using a one-way ANOVA. Effect of propionibacteria consumption on this parameter was assessed by comparing each group at initial $v$. final time using a paired Student's $t$ test, and by comparing groups in pairs at the end of the experiment using a one-way ANOVA followed by a Student-Newman-Keuls multiple comparison test. Statistical significance was set at $P<0 \cdot 05$. Calculations were performed using the Statview ${ }^{\circledR}$ software (version 5.0; SAS Institute, Cary, NC, USA). All data were expressed as mean values with their standard errors $(n 6)$.

Table 4. Oligonucleotidic probes used in fluorescent in situ hybridisation analysis

\begin{tabular}{|c|c|c|c|}
\hline Probes & Sequences from $5^{\prime}$ to $3^{\prime *}$ & OPD code & Reference \\
\hline Eub338 & GCTGCCTCCCGTAGGAGT & S-D-Bact-0338-a-A-18 & Amann et al. (1995) \\
\hline Non-Eub338 & ACATCCTACGGGAGGC & NA & Wallner et al. (1993) \\
\hline Ato291 & GGTCGGTCTCTCAACCC & S-«-Ato-0291-a-A-17 & Harmsen et al. (2000) \\
\hline Вас303 & CCAATGTGGGGGACCTT & S---Bacto-0303-a-A-17 & Manz et al. (1996) \\
\hline Bif164 & CATCCGGCATTACCACCC & S-G-Bif-0164-a-A-18 & Langendijk et al. (1995) \\
\hline Clep866 & GGTGGATWACTTATTGTG & S----Clep-0866-a-A-18 & Lay et al. $(2005 b)$ \\
\hline Clep866 competitor $1 \dagger$ & GGTGGAAWACTTATTGTG & & \\
\hline Clep866 competitor $2 \dagger$ & GGTGGATWACTTATTGCG & & \\
\hline Erec482 & GCTTCTTAGTCARGTACCG & S---Erec-0482-a-A-19 & Franks et al. (1998) \\
\hline Enter1432 & CTTTTGCAACCCACT & S---Ent-1432-a-A-15 & Sghir et al. (2000) \\
\hline Lab158 & GGTATTAGCAYCTGTTTCCA & S-- -Lab-0158-a-A-20 & Harmsen et al. (1999) \\
\hline
\end{tabular}

OPD, Oligonucleotide Probe Database.

${ }^{*} \mathrm{R}$ is an $\mathrm{A}$ or $\mathrm{G}, \mathrm{Y}$ is a $\mathrm{C}$ or $\mathrm{T}$ and $\mathrm{W}$ is an $\mathrm{A}$ or $\mathrm{T}$.

†Targets of the Clep866 oligonucleotide competitors 1 and 2 are Sb. termiti and AF001743 respectively; the names correspond to RDP Short-ID (Lay et al. 2005b). 


\section{Results}

Tolerance of dairy propionibacteria towards digestive stresses and abilities for short-chain fatty acid production in vitro

With the goal to select propionibacteria candidates for in vivo assays, twenty-four strains of $P$. freudenreichii and one of $P$. acidipropionici, isolated either from dairy products or from human faecal microbiota, were tested in vitro, on the one hand according to their resistance to digestive stresses and, on the other hand, according to propionate production ability in a medium mimicking the human colonic content. The strain survival was measured following sequential exposures to acidic $\mathrm{pH}$ and ox bile at $37^{\circ} \mathrm{C}$. Results are represented in Fig. 1 (A), strains being classified by decreased order of survival. Digestive stress tolerance was highly variable, depending on the strain. The strains showing the best rate of survival during digestive stress challenge, that is to say the strains that underwent a viability loss below 1-log value, are four $P$. freudenreichii shermanii strains, TL133, TL1348, TL213 and TL1123, and one $P$. freudenreichii freudenreichii strain, TL3. Most of the strains showed a moderate loss of viability between 1- and 2-log values, whereas four out of the twenty-five strains (TL33, TL166, TL165 and TL2) were shown to be the less tolerant strains and underwent a drop in viability greater than 2-log values.

With the aim to select the best SCFA-producing strains, propionibacteria were cultured in Gibson modified medium at $37^{\circ} \mathrm{C}$ and propionate production was quantified after $24 \mathrm{~h}$ incubation (Fig. 1 (B)). A set of strains produced an amount of propionate close to the $1.32 \mathrm{~g} / \mathrm{l}$ value predicted for the lactate provided $(3 \mathrm{~g} / \mathrm{l})$ to be completely metabolised. Twelve
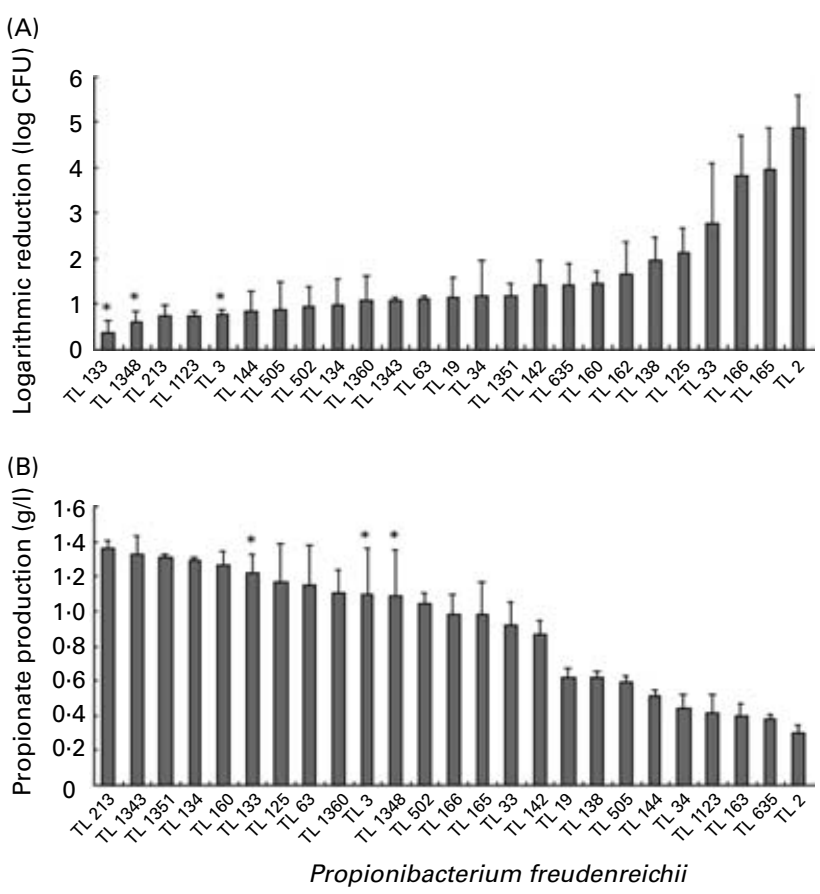

Fig. 1. In vitro screening of Propionibacterium strains: resistance to digestive stress (A) and production of propionate (B). (A) Viability reduction of twentyfive strains after acid challenge followed by bile challenge. (B) Production of propionate after $24 \mathrm{~h}$ of culture in a medium mimicking the content of the human colon. Data are means with their standard errors represented by vertical bars. * The three best candidates selected for in vivo experiments. strains showed thus a propionate production above $1 \mathrm{~g} / 1$, including TL213, TL133, TL3 and TL1348. By contrast, a set of strains produced lower amounts of propionate. All the tested strains led to comparable concentrations of propionate after $3 \mathrm{~d}$ of incubation, showing that the rate, but not the yield, of propionic fermentation varied (data not shown).

It is noteworthy that both digestive stress tolerance and propionate production rate are strain-dependent. These screenings allowed us to select three strains for in vivo assays, two $P$. freudenreichii subsp. shermanii (TL133 and TL1348) and one $P$. freudenreichii subsp. freudenreichii (TL3), which fulfilled the two selection criteria.

\section{Survival of selected strains of propionibacteria in the gastrointestinal tract of rats}

As shown in Table 5, no dairy propionibacteria could be detected in rats' faeces at the beginning of the experiment. This remained true in control animals throughout the experiment. In groups consuming propionibacteria, recovery varied according to the strain and the time of analysis. Indeed, after $10 \mathrm{~d}$ of propionibacteria administration (medium time), faecal concentration did not exceed 6-log values per $\mathrm{g}$ for the TL3 strain whereas it reached 8-log values for the TL133 and TL1348 strains. This 2-5-log difference in terms of survival was minimised during further propionibacteria feeding. Hence, after another $10 \mathrm{~d}$, about 1-log difference was observed between the TL3 group and the other TL groups. Indeed, the dairy propionibacteria level in the faeces increased to a 7-log value per $g$ in the TL3 group, whereas it remained constant until the end of the experiment (final time) in the TL133 and TL1348 groups. Surprisingly, at the end of the experiment, caecal concentrations of the three strains were very similar. Indeed, close to 8-log of propionibacteria per $g$ were enumerated in the caecal contents of rats that had consumed propionibacteria, regardless of the strain (Table 5).

\section{Metabolic activity of selected strains of propionibacteria in the gastrointestinal tract of rats}

With the aim of examining if strains were metabolically active in the rat digestive tract, expression of the gene encoding methylmalonyl-CoA carboxyltransferase, a specific and key enzyme of $P$. freudenreichii metabolism, was analysed using reverse transcription PCR. Transcarboxylase mRNA was detected in the caecal contents of all rats consuming propionibacteria (threshold cycles 27.2 (SEM 0.2); 26.8 (SEM 0.5) and $26 \cdot 2$ (SEM 0.2) for the TL3 ( $n$ 4), TL133 ( $n$ 5) and TL1348 ( $n$ 4) groups respectively), regardless of the strain, whereas no expression of this transcript was detected in the control group (n 5).

\section{Effect of propionibacteria consumption on caecal $\mathrm{pH}$, short-chain fatty acids and enzymic activities}

First, it is noticeable that rats' health remained good throughout the experiment, whether the animals belonged to the control or TL groups. Consumption of propionibacteria had no significant effect on weight gain (25 (SEM 2) g/rat); consequently, rats' body weight at the end of the experiment was 
Table 5. Enumeration of dairy propionibacteria in faeces and caecal contents of rats fed with Propionibacterium freudenreichii TL3, TL133 or TL1348 strains for $20 \mathrm{~d} \dagger$

(Mean values with their standard errors for six rats per group)

\begin{tabular}{|c|c|c|c|c|c|c|c|}
\hline & \multirow{2}{*}{$\begin{array}{c}\text { Control }(\log \text { CFU/g) } \\
\text { Mean }\end{array}$} & \multicolumn{2}{|c|}{$\begin{array}{c}\text { TL3 } \\
(\log \mathrm{CFU} / \mathrm{g})\end{array}$} & \multicolumn{2}{|c|}{$\begin{array}{c}\text { TL133 } \\
(\log \text { CFU/g) }\end{array}$} & \multicolumn{2}{|c|}{$\begin{array}{c}\text { TL1348 } \\
(\log \text { CFU/g) }\end{array}$} \\
\hline & & Mean & SEM & Mean & SEM & Mean & SEM \\
\hline \multicolumn{8}{|l|}{ Faeces } \\
\hline Initial time (day 21) & ND & ND & & ND & & ND & \\
\hline Medium time (day 31 ) & ND & $5 \cdot 78^{\star}$ & 0.49 & $8 \cdot 22$ & 0.42 & 8.51 & 0.78 \\
\hline Final time (day 41) & ND & $6 \cdot 69^{\star}$ & 0.60 & 7.92 & 0.29 & 8.37 & 0.54 \\
\hline Caecal contents (day 42) & ND & $7 \cdot 81$ & 0.42 & $7 \cdot 75$ & 0.29 & 8.04 & 0.28 \\
\hline
\end{tabular}

the same among groups (316 (SEM 4) g). Food consumption was also similar in all groups, ranging from $2.6 \mathrm{~kg}(n 6$; $20 \mathrm{~d})$ in the TL3 group to $3.2 \mathrm{~kg}(n 6 ; 20 \mathrm{~d})$ in the control group. No significant differences in caecal weight and $\mathrm{pH}$ were observed between groups (Table 6).

By contrast, the total SCFA concentration was significantly enhanced in the caecal content of rats receiving the TL133P. freudenreichii strain compared with the three other groups with a significant increase of acetate, propionate and butyrate (Table 6). Nevertheless, the relative proportions of acetate, propionate and butyrate did not differ between groups; they were 77.8 (SEM 0.3), 13.2 (SEM 0.3) and 9.0 (SEM 0.2) \%, respectively. With regard to minor SCFA, consumption of propionibacteria significantly decreased the concentration of branched-chain fatty acids, regardless of the strain.

$\beta$-Galactosidase and $\beta$-glucuronidase activities were measured in the faecal contents at the end of the experiment. No significant difference was observed between groups, either for $\beta$-galactosidase activity (6.6 (SEM 0.4) $\mu \mathrm{mol} / \mathrm{min}$ per g), or for $\beta$-glucuronidase activity (1.5 (SEM $0 \cdot 1) \mu \mathrm{mol} / \mathrm{min}$ per $\mathrm{g}$ ).

\section{Effect of propionibacteria consumption on the faecal microbiota diversity}

The impact of dairy propionibacteria consumption on the faecal microbiota composition was assessed by FISH combined with flow cytometry at the start of the experiment, i.e. 3 weeks after all initially germ-free rats had been inoculated with the human faecal microbiota. No significant difference was observed between groups, indicating that the microbiota composition was basically the same in all rats before propionibacteria administration (Fig. 2 (A)). The most abundant clusters were $C$. coccoides $-E$. rectale, detected with the Erec482 probe (Table 4), and C. leptum, detected with the Clep866 probe; they represented $35 \cdot 3$ (SEM 0.6) and 30.2 (SEM 0.8 ) $\%$ of the total eubacteria, respectively. The Bacteroides group, detected with the Bac 303 probe, accounted for 25.5 (SEM 1.0) \% of the bacterial population and the Bifidobacterium group, detected with the Bif164 probe, was the fourth major group $(6 \cdot 2$ (SEM $0 \cdot 2) \%)$. The four other phylogenetic groups, i.e. Atopobium, enterobacteria and Lactobacillus-Enterococcus, accounted together for less than $5 \%$ of the total. After 3 weeks of propionibacteria administration, analysis of the faecal microbiota was performed again (Fig. 2 (B)). The proportion of the Bacteroides cluster had significantly increased in the TL133 group (27.2 (SEM 1.1) \% at initial time $v .35 .6$ (SEM 2.8) \% at final time), whereas it remained stable in the other groups. Similarly, rats from the TL1348 group harboured a slightly higher proportion of enterobacteria at the end of the experiment (1.8 (SEM 0.2) \% at initial time $v .3 \cdot 2$ (SEM 0.4$) \%$ at final time), though it remained in the same low order of magnitude as the other

Table 6. Effect of TL3, TL133 and TL1348 supplementation on the weight, pH and short-chain fatty acid concentrations of the caecal contents

(Mean values with their standard errors for six rats per group)

\begin{tabular}{|c|c|c|c|c|c|c|c|c|}
\hline & \multicolumn{2}{|c|}{ Control } & \multicolumn{2}{|c|}{ TL3 } & \multicolumn{2}{|c|}{ TL133 } & \multicolumn{2}{|c|}{ TL1348 } \\
\hline & Mean & SEM & Mean & SEM & Mean & SEM & Mean & SEM \\
\hline Weight (g) & $5 \cdot 80$ & 0.43 & 3.86 & 0.60 & $5 \cdot 72$ & 0.24 & 5.56 & 0.39 \\
\hline $\mathrm{PH}$ & $6 \cdot 54$ & 0.07 & 6.59 & 0.06 & 6.58 & $0 \cdot 11$ & 6.49 & 0.05 \\
\hline Acetate $(\mu \mathrm{mol} / \mathrm{g})$ & $30 \cdot 14$ & $4 \cdot 19$ & 29.85 & 1.30 & $39 \cdot 61^{*}$ & 1.49 & 25.03 & 3.23 \\
\hline Propionate $(\mu \mathrm{mol} / \mathrm{g})$ & $5 \cdot 23$ & 0.67 & 4.70 & 0.31 & $6 \cdot 80^{*}$ & 0.19 & 4.22 & 0.46 \\
\hline Butyrate $(\mu \mathrm{mol} / \mathrm{g})$ & 3.34 & 0.56 & 3.54 & 0.25 & $4 \cdot 80^{\star}$ & 0.30 & $2 \cdot 87$ & 0.37 \\
\hline Valerate and caproate $(\mu \mathrm{mol} / \mathrm{g})$ & 1.03 & 0.10 & 0.71 & 0.06 & 0.73 & $0 \cdot 11$ & 0.74 & 0.13 \\
\hline Branched-chain fatty acids $(\mu \mathrm{mol} / \mathrm{g})$ & $0.38^{*}$ & 0.06 & 0.19 & 0.03 & 0.23 & 0.01 & 0.24 & 0.03 \\
\hline Total SCFA $(\mu \mathrm{mol} / \mathrm{g})$ & $40 \cdot 12$ & 5.41 & 38.99 & 1.79 & $52 \cdot 17^{\star}$ & 1.67 & $33 \cdot 10$ & 4.12 \\
\hline
\end{tabular}

* Mean value was significantly different from those of the other groups (ANOVA and Student-Newman-Keuls test; $P<0.05$ ). 

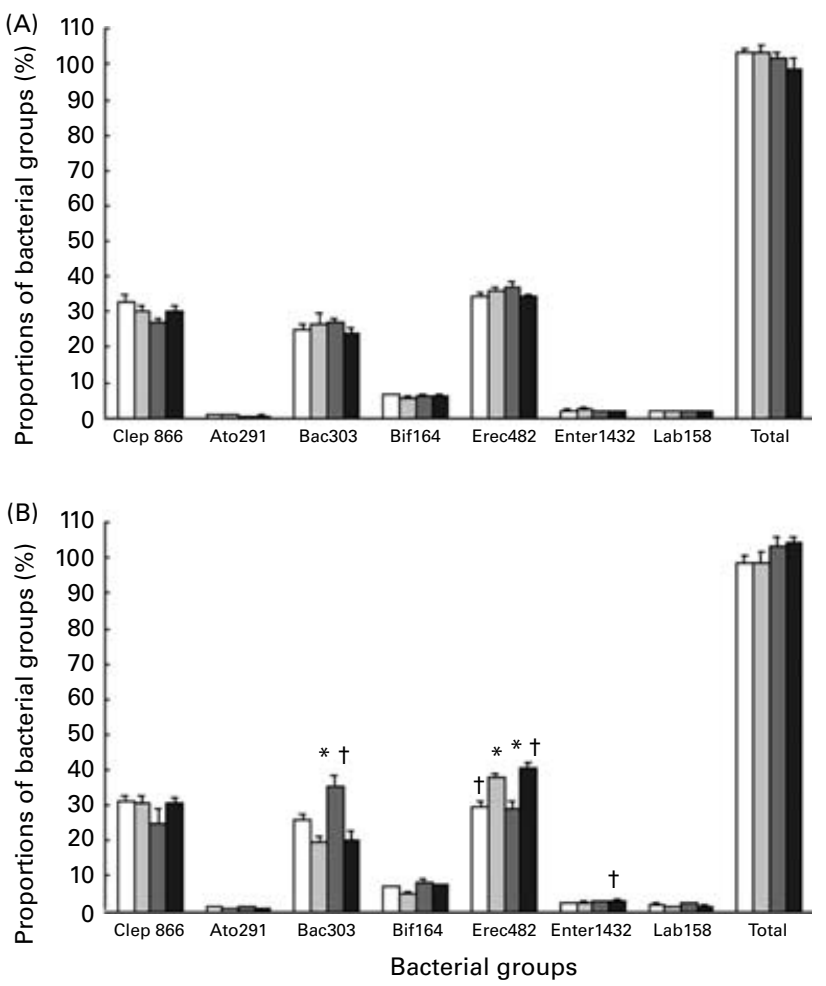

Fig. 2. Analysis of faecal microbiota of rats before (A) and after (B) a $20 \mathrm{~d}$ consumption period of Propionibacterium freudenreichii TL133 ( $\square$ ), TL1348 $(\square)$ or TL3 $(\square)$ strain, and for control rats $(\square)$. Proportions of Clostridium leptum subgroup (Clep866), Atopobium (Ato291), Bacteroides (Bac303), Bifidobacterium (Bif 164), C. coccoides-Eubacterium rectale (Erec482), Enterics (Enter1432) and Lactobacilli-Enterococci (Lab158) groups in control rats and propionibacteria-supplemented rats were assessed in the faeces using fluorescent in situ hybridisation combined with flow cytometry. Data are means $(n 6)$ with their standard errors represented by vertical bars and are the proportions of cells hybridising with a specific probe among cells hybridising with the universal Eub338 probe. * Mean value was significantly different from that of the control group at the same time (ANOVA and Student-Newman-Keuls test; $P<0.05)$. $†$ Mean value was significantly different from that of the same group at initial time (paired Student's $t$ test; $P<0.05$ ).

groups. Finally, the proportion of the $C$. coccoides $-E$. rectale cluster spontaneously decreased with time in the control group (34.1 (SEM 1.4) \% at initial time v. 29.8 (SEM 1.2) \% at final time) whereas it did not change in the TL3 and TL133 groups and increased in the TL1348 group (34.1 (SEM 0.6) $\%$ at initial time $v .40 \cdot 3$ (SEM 1.8) \% at final time). On the whole, at the end of the experiment, the proportion of the $C$. coccoides $-E$. rectale cluster appeared to be higher in the TL3 and TL1348 groups than in the control and TL133 groups.

\section{Discussion}

Probiotic potential has been described for a limited number of dairy Propionibacterium strains and mainly depends on the ability (1) to survive and reach substantial populations within the digestive tract and (2) to maintain a metabolic activity in the colon. The selection of strains of $P$. freudenreichii best adapted for survival in the gut is a prerequisite to the development of an efficient probiotic product.

The present study showed a tremendous variability in the probiotic abilities, as revealed in vitro. The criteria of screening chosen were tolerance towards digestive stresses and rate of SCFA synthesis in conditions mimicking the intestinal environment $\left(37^{\circ} \mathrm{C}\right.$, artificial intestinal content medium). The first criterion, acid and bile stress, is supposed to reflect the robustness of the strain in the adverse environment of the human digestive tract and is frequently considered. Variability among strains of propionibacteria was already observed during acid stress (Jan et al. 2000) and bile stress (WarminskaRadyko et al. 2002). However, while acid adaptation was shown to trigger bile salts sensitivity (Leverrier et al. 2004), the succession of both stresses, which is more stringent, was not taken into account for propionibacteria strain selection. The second criterion would reflect the ability to maintain a metabolic activity, SCFA propionate being one of the endproducts of propionibacteria obligatory fermentative metabolism. Tremendous differences in performance were observed, suggesting that not all the isolated strains have a probiotic potential. On one hand, the in vitro method can be applied to a large number of strains in order to expand screening. On the other hand, screenings have to be confirmed by in vivo investigations, as in vitro challenges are far from the conditions prevailing in the digestive tract.

In the present study, survival of the three strains of P. freudenreichii selected in vitro was demonstrated in vivo, and high-level populations were detected in the intestine of human microbiota-associated rats. These animals constitute a now well-validated model for experimental studies aimed at evaluating the effects of functional foods, including probiotics, in gastrointestinal physiology (Djouzi et al. 1997; Kleessen et al. 2001; Humblot et al. 2004; Imaoka et al. 2004). Indeed, human microbiota inoculated to germ-free rats colonise the foreign gastrointestinal tract while retaining their major characteristics in terms of bacterial populations, enzyme activities and metabolite profiles (Andrieux et al. 1991; Rumney \& Rowland, 1992). Furthermore this model facilitates investigations on the interactions between food compounds and the human gut microbiota, since it is easier to control animal diets than those of human subjects. In addition, it enables several repetitions of the same microbiota for one dietary condition, thus avoiding the fluctuations observed by several authors in human experiments (Tannock, 2001; Zoetendal et al. 2001). Finally, this model allows using invasive investigations in order to evaluate propionibacteria metabolic activity in situ. Since SCFA are absorbed by the colonic mucosa, their quantification within the intestine is essential, while their concentration in faeces may not reflect their production rate.

Despite the possible competition with the human microbiota, ingested propionibacteria reached populations between $10^{7}$ and $10^{8} \mathrm{CFU} / \mathrm{g}$ in faeces after several days of administration. As a comparison, another dairy propionibacterium, $P$. jensenii, reached a concentration of $10^{8}$ to $10^{9} \mathrm{CFU} / \mathrm{g}$ in faeces of rats following administration at a daily dose of $10^{10} \mathrm{CFU}$ (Huang et al. 2003), in agreement with the present results. While similar populations of $P$. freudenreichii (close to $10^{8} \mathrm{CFU} / \mathrm{g}$ ) were found for each TL strain in the rats' caecal contents, up to $1-\log$ value less of viable cells were detected in the faeces than in the caeca of rats consuming the TL3 strain. A possible explanation for TL3 viability drop could be a particular sensitivity of this strain to hydric stress due to massive water absorption in the colon. Indeed, 
the efficacy of osmoadaptation is highly strain-dependent within the P. freudenreichii species (Boyaval et al. 1999; Cardoso et al. 2004). TL3 may also be more sensitive to the barrier effect of the human microbiota. The minimal level allowing a micro-organism to influence the environment is generally recognised as $10^{6}$ to $10^{8} \mathrm{CFU} / \mathrm{g}$ intestinal content (Marteau et al. 1993). The caecal and faecal propionibacteria populations reached in the present study by TL133 and TL1348 thus suggest that they may have had an impact on the colon ecosystem and/or metabolism.

The metabolic activity of propionibacteria during transit in the digestive tract remains a key question. To evaluate it, a molecular method based on the specific detection of the mRNA encoding the propionibacteria transcarboxylase within the complex intestinal microbiota was used (Hervé et al. 2006). This protein, which contains sequences strictly specific to dairy propionibacteria, is a key enzyme of the transcarboxylase cycle involved in propionic fermentation. Transcription of transcarboxylase occurs during fermentation and stops in late stationary phase when starvation occurs. The corresponding mRNA is thus considered as a biomarker of metabolic activity (Hervé et al. 2006). The results obtained in the present study demonstrate that the three $P$. freudenreichii strains initiate transcarboxylase gene transcription during transit as the mRNA is detected in the caecal content of propionibacteria-supplemented rats, yet undetected in that of control rats. It can thus be hypothesised that these bacteria encountered conditions allowing active metabolism during transit in the digestive tract. Propionic fermentation leads to the production of the SCFA acetate and propionate. However, a very high proportion of SCFA is taken up by the colonic mucosa, which is why SCFA are preferentially quantified in the caecum rather than in the faeces. This was investigated in propionibacteria-supplemented and control human microbiota-associated rats. The overall SCFA caecal concentration slightly increased as a result of TL133 ingestion, while TL3 and TL1348 failed to exert such an effect. Acetate, propionate and butyrate concentrations were enhanced, while branched-chain fatty acids were lowered. A modification in both the amount and the composition of caecal SCFA was reported as a result of ingestion of milk containing $P$. acidipropionici or $P$. freudenreichii in mice (Perez Chaia \& Zarate, 2005). Furthermore, in a human study using a commercially available $P$. freudenreichii probiotic preparation, the recovery of live propionibacteria seemed to be related to an enhanced content in SCFA and a lower branched-chain fatty acid ratio, in faecal samples (Jan et al. 2002b). Moreover, a lower branched-chain fatty acid ratio was also observed as a result of yoghurt consumption by infants (Guerin-Danan et al. 1998). As suggested by the authors, the decrease in these fatty acids, characteristic of bacterial proteolytic reactions which lead to the production of toxic metabolites, may provide a healthful influence on the host. In the present study, the P. freudenreichii TL133 strain may thus have exerted a beneficial influence on the intestinal metabolism.

Other effects on the gut ecosystem were further sought during the present study. No significant variations in the colon $\beta$-galactosidase and $\beta$-glucuronidase enzymic activities occurred during propionibacteria ingestion. In contrast, it has been reported that ingestion of $P$. acidipropionici increases $\beta$-galactosidase and decreases $\beta$-glucuronidase activities in the caecum of mice (Perez Chaia et al. 1999; Perez Chaia \&
Zarate, 2005). However, such an effect was not observed in the caecal contents of rats neither during the present study, nor with $P$. jensenii in rat faeces (Huang et al. 2003). These results seem to confirm that modulation of this bacterial enzyme activity is species-dependent. No major modification of the gut microbial ecology occurred in rats consuming the propionibacteria. Using cluster-targeting probes, the microbial composition of faecal samples was analysed by FISH combined with flow cytometry; this method estimates the relative proportion of a bacterial group within the total number of bacteria. The Bacteroides group, which is predominant in the human faecal microbiota, was increased in rats fed with the TL133 strain. However, this population increase remained within the proportion range from 0.0 to $47.9 \%$ of Bacteroides cells obtained in a human study involving twenty subjects (Rigottier-Gois et al. 2003b). By contrast, the P. freudenreichii TL1348 strain increased the faecal population of $C$. coccoides $-E$. rectale to a proportion (40.3\%) slightly exceeding the maximum value $(38.5 \%)$ observed by Rigottier-Gois et al. (2003a) among twenty-five human subjects; nevertheless, these values are fully compatible with the proportions observed by Lay et al. (2005b)in a group of twenty-one subjects or in the 100 subjects they analysed for a large-scale molecular analysis of the normal colonic microbiota in healthy human subjects from Northern Europe (Lay et al. 2005a). No modification of the bifidobacteria population was detected, in contrast with other reports. Indeed, a bifidogenic effect was observed in vitro with cell-free extracts of $P$. freudenreichii (Kaneko et al. 1994), due to the presence of bifidogenic compounds (Mori et al. 1997; Isawa et al. 2002). Similarly, ingestion of $P$. freudenreichii, under the form of whey cultures, either heat-inactivated (Satomi et al. 1999) or not (Hojo et al. 2002), or as freeze-dried live bacteria (Bougle et al. 1999), resulted in a higher faecal bifidobacteria concentration in human subjects. It must be stressed that the bifidobacteria proportion in the human microbiota used in the present study was high, about $6 \%$, compared with the $2 \%$ average values obtained in the human studies performed (Lay et al. $2005 a, b)$. Furthermore, the bifidogenic property would depend on the strain within the $P$. freudenreichii species $(\mathrm{N}$ Roland, personal communication). On the whole, the present study shows that modification of the colon ecosystem by propionibacteria is limited and strain-specific.

To summarise, three strains of $P$. freudenreichii, firstly selected in vitro on the basis of stress challenges and metabolic assays, are able to survive at high population levels and to be metabolically active in the gastrointestinal tract of human microbiota-associated rats. Furthermore, while no drastic effect on the rats' physiology was observed as a result of propionibacteria ingestion, strains modulated differently the intestinal microbiota composition and SCFA content. The present study thus confirms the need for large-scale screenings of dairy propionibacteria, involving simple in vitro assays followed by in vivo validation experiments, to reveal the probiotic abilities of specific strains. Here, one particular strain, namely TL133, displayed an interesting probiotic potential by increasing the caecal concentration of major SCFA while decreasing branched-chain fatty acids. Colonic SCFA, produced by bacterial fermentation of dietary fibre and starch in the large bowel, are known for their beneficial impact on colonic health, including development of normal colonic 
epithelial cells and protection against cancer and ulcerative colitis (Hague et al. 1997). Furthermore, propionibacterial SCFA are potent inducers of apoptosis in colon cancer cells in vitro (Jan et al. 2002a; Lan et al. in press). The effects of a selected strain of $P$. freudenreichii on the intestinal microbial ecosystem and metabolism, which would lead to an enhanced production of major SCFA in vivo, opens further perspectives for the probiotic application of dairy propionibacteria. The same in vivo model should allow studying the impact of selected strains of dairy propionibacteria on experimental colon carcinogenesis.

\section{Acknowledgements}

The authors thank Rosa Durao for breeding the germ-free rats, Alhosna Benjdia and Marc Fondevrez for their technical assistance with FISH analysis and RNA extraction, respectively. The authors acknowledge Laboratoires Standa for financial support and for a constant interest. The present study has been supported by a grant from CRITT Santé Bretagne. The authors thank Joseph Kehoe for English correction.

\section{References}

Amann RI, Ludwig W \& Schleifer KH (1995) Phylogenetic identification and in situ detection of individual microbial cells without cultivation. Microbiol Rev 59, 143-169.

Anastasiou R, Leverrier P, Krestas I, Rouault A, Kalantzopoulos G, Boyaval P, Tsakalidou E \& Jan G (2006) Changes in protein synthesis during thermal adaptation of Propionibacterium freudenreichii subsp, shermanii. Int J Food Microbiol 108, 301-314.

Andrieux C, Hibert A, Houari AM, Bensaada M, Popot F \& Szylit O (1998) Ulva lactuca is poorly fermented but alters bacterial metabolism in rats inoculated with human faecal flora from methane and non-methane producers. J Sci Food Agric 77, 25-30.

Andrieux C, Lory S, Dufour-Lescoat C, de Baynast R \& Szylit O (1991) Physiological effects of inulin in germ-free rats and in heteroxenic rats inoculated with a human flora. Food Hydrocolloids $\mathbf{5}$, $49-56$.

Bougle D, Roland N, Lebeurrier F \& Arhan P (1999) Effect of propionibacteria supplementation on fecal bifidobacteria and segmental colonic transit time in healthy human subjects. Scand $J$ Gastroenterol 34, 144-148.

Boyaval P, Deborde C, Corre C, Blanco C \& Begue E (1999) Stress and osmoprotection in propionibacteria. Lait 79, 59-69.

Cardoso FS, Gaspar P, Hugenholtz J, Ramos A \& Santos H (2004) Enhancement of trehalose production in dairy propionibacteria through manipulation of environmental conditions. Int J Food Microbiol 91, 195-204.

Coates ME (1968) The Germ-free Animal in Research. London and New York: Academic Press.

Djouzi Z, Andrieux C, Degivry MC, Bouley C \& Szylit O (1997) The association of yogurt starters with Lactobacillus casei DN 114.001 in fermented milk alters the composition and metabolism of intestinal microflora in germ-free rats and in human flora-associated rats. J Nutr 127, 2260-2266.

Food and Agriculture Organization \& World Health Organization (2002) Guidelines for the Evaluation of Probiotics in Food. Geneva: FAO/WHO Working Group Report.

Franks AH, Harmsen HJ, Raangs GC, Jansen GJ, Schut F \& Welling GW (1998) Variations of bacterial populations in human feces measured by fluorescent in situ hybridization with group-specific 16S rRNA-targeted oligonucleotide probes. Appl Environ Microbiol 64, 3336-3345.
Gibson GR \& Wang X (1994) Enrichment of bifidobacteria from human gut contents by oligofructose using continuous culture. FEMS Microbiol Lett 118, 121-127.

Guerin-Danan C, Chabanet C, Pedone C, Popot F, Vaissade P, Bouley C, Szylit O \& Andrieux C (1998) Milk fermented with yogurt cultures and Lactobacillus casei compared with yogurt and gelled milk: influence on intestinal microflora in healthy infants. Am J Clin Nutr 67, 111-117.

Hague A, Singh B \& Paraskeva C (1997) Butyrate acts as a survival factor for colonic epithelial cells: further fuel for the in vivo versus in vitro debate. Gastroenterology 112, 1036-1040.

Harmsen HJ, Elfferich P, Schut F \& Welling GW (1999) A $16 \mathrm{~S}$ rRNA-targeted probe for detection of lactobacilli and enterococci in faecal samples by fluorescent in situ hybridization. Microbial Ecol Health Dis 11, 3-12.

Harmsen HJ, Wildeboer-Veloo AC, Grijpstra J, Knol J, Degener JE \& Welling GW (2000) Development of 16S rRNA-based probes for the Coriobacterium group and the Atopobium cluster and their application for enumeration of Coriobacteriaceae in human feces from volunteers of different age groups. Appl Environ Microbiol 66, 4523-4527.

Hervé C, et al. (2006) Transcarboxylase mRNA: a marker which evidences $P$. freudenreichii survival and metabolic activity during its transit in the human gut. Int J Food Microbiol. doi:10.1016/ j.ijfoodmicro.2006.08.013.

Hojo K, Yoda N, Tsuchita H, Ohtsu T, Seki K, Taketomo N, Murayama T \& Lino H (2002) Effect of ingested culture of Propionibacterium freudenreichii ET-3 on fecal microflora and stool frequency in healthy females. Biosci Microflora 21, 115-120.

Holo H, Faye T, Brede DA, Nilsen T, Odegard I, Langsrud T, Brendehaug J \& Nes IF (2002) Bacteriocins of propionic acid bacteria. Lait 82, 59-68.

Huang Y, Kotula L \& Adams MC (2003) The in vivo assessment of safety and gastrointestinal survival of an orally administered novel probiotic, Propionibacterium jensenii 702, in a male Wistar rat model. Food Chem Toxicol 41, 1781-1787.

Hugenholtz J, Hunik J, Santos H \& Smid E (2002) Nutraceutical production by propionibacteria. Lait 82, 103-112.

Humblot C, Lhoste E, Knasmuller S, Gloux K, Bruneau A, Bensaada M, Durao J, Rabot S, Andrieux C \& Kassie F (2004) Protective effects of Brussels sprouts, oligosaccharides and fermented milk towards 2-amino-3-methylimidazo[4,5-f]quinoline (IQ)-induced genotoxicity in the human flora associated F344 rat: role of xenobiotic metabolising enzymes and intestinal microflora. J Chromatogr 802B, 231-237.

Imaoka A, Setoyama H, Takagi A, Matsumoto S \& Umesaki Y (2004) Improvement of human faecal flora-associated mouse model for evaluation of the functional foods. J Appl Microbiol 96, 656-663.

Isawa K, Hojo K, Yoda N, et al. (2002) Isolation and identification of a new bifidogenic growth stimulator produced by Propionibacterium freudenreichii ET-3. Biosci Biotechnol Biochem 66, 679-681.

Jan G, Belzacq AS, Haouzi D, Rouault A, Metivier D, Kroemer G \& Brenner C (2002a) Propionibacteria induce apoptosis of colorectal carcinoma cells via short-chain fatty acids acting on mitochondria. Cell Death Differ 9, 179-188.

Jan G, Leverrier P \& Roland N (2002b) Survival and beneficial effects of propionibacteria in the human gut: in vivo and in vitro investigations. Lait 82, 131-144.

Jan G, Rouault A \& Maubois JL (2000) Acid stress susceptibility and acid adaptation of Propionibacterium freudenreichii subsp. shermanii. Lait 80, 325-336.

Kaneko T, Mori H, Iwata M \& Meguro S (1994) Growth stimulator for bifidobacteria produced by Propionibacterium freudenreichii and several intestinal bacteria. J Dairy Sci 77, 393-404.

Kirjavainen PV, El Nezami HS, Salminen SJ, Ahokas JT \& Wright PF (1999) Effects of orally administered viable Lactobacillus 
rhamnosus GG and Propionibacterium freudenreichii subsp. shermanii JS on mouse lymphocyte proliferation. Clin Diagn Lab Immunol 6, 799-802.

Kleessen B, Hartmann L \& Blaut M (2001) Oligofructose and long-chain inulin: influence on the gut microbial ecology of rats associated with a human faecal flora. Br J Nutr 86, 291-300.

Lan A, Lagadic-Gossmann D, Lemaire C, Brenner C \& Jan G (in press) Acidic extracellular $\mathrm{pH}$ shifts colorectal cancer cell death from apoptosis to necrosis upon exposure to propionate and acetate, major end-products of the human probiotic propionibacteria. Apoptosis.

Langendijk PS, Schut F, Jansen GJ, Raangs GC, Kamphuis GR, Wilkinson MH \& Welling GW (1995) Quantitative fluorescence in situ hybridization of Bifidobacterium spp. with genus-specific $16 \mathrm{~S}$ rRNA-targeted probes and its application in fecal samples. Appl Environ Microbiol 61, 3069-3075.

Lay C, Rigottier-Gois L \& Holmstrom K, et al. (2005a) Colonic microbiota signatures across five northern European countries. Appl Environ Microbiol 71, 4153-4155.

Lay C, Sutren M, Rochet V, Saunier K, Dore J \& Rigottier-Gois L (2005b) Design and validation of $16 \mathrm{~S}$ rRNA probes to enumerate members of the Clostridium leptum subgroup in human faecal microbiota. Environ Microbiol 7, 933-946.

Leverrier P, Dimova D, Pichereau V, Auffray Y, Boyaval P \& Jan G (2003) Susceptibility and adaptive response to bile salts in Propionibacterium freudenreichii: physiological and proteomic analysis. Appl Environ Microbiol 69, 3809-3818.

Leverrier P, Vissers JP, Rouault A, Boyaval P \& Jan G (2004) Mass spectrometry proteomic analysis of stress adaptation reveals both common and distinct response pathways in Propionibacterium freudenreichii. Arch Microbiol 181, 215-230.

Lyon WJ, Sethi JK \& Glatz BA (1993) Inhibition of psychrotrophic organisms by propionicin PLG-1, a bacteriocin produced by Propionibacterium thoenii. J Dairy Sci 76, 1506-1513.

Malik AC, Reinbold GW \& Vedamuthu ER (1968) An evaluation of the taxonomy of Propionibacterium. Can J Microbiol 14, $1185-1191$.

Manz W, Amann R, Ludwig W, Vancanneyt M \& Schleifer KH (1996) Application of a suite of 16S rRNA-specific oligonucleotide probes designed to investigate bacteria of the phylum cytophagaflavobacter-bacteroides in the natural environment. Microbiology 142, 1097-1106.

Marteau P, Pochart P, Bouhnik Y \& Rambaud JC (1993) The fate and effects of transiting, nonpathogenic microorganisms in the human intestine. World Rev Nutr Diet 74, 1-21.

Marteau PR, de Vrese M, Cellier CJ \& Schrezenmeir J (2001) Protection from gastrointestinal diseases with the use of probiotics. Am J Clin Nutr 73, Suppl. 2, 430S-436S.

Michel C, Roland N, Lecannu G, Hervé C, Avice JC, Rival M \& Cherbut C (2005) Colonic infusion with Propionibacterium acidipropionici reduces severity of chemically-induced colitis in rats. Lait 85, 99-111.

Mori H, Sato Y, Taketomo N, Kamiyama T, Yoshiyama Y, Meguro S, Sato H \& Kaneko T (1997) Isolation and structural identification of bifidogenic growth stimulator produced by Propionibacterium freudenreichii. J Dairy Sci 80, 1959-1964.

Mortensen PB \& Clausen MR (1996) Short-chain fatty acids in the human colon: relation to gastrointestinal health and disease. Scand J Gastroenterol 216, Suppl., 132-148.
Ouwehand AC, Salminen S \& Isolauri E (2002) Probiotics: an overview of beneficial effects. Antonie Van Leeuwenhoek 82, 279-289.

Perez Chaia A, de Macias MEN \& Oliver G (1995) Propionibacteria in the gut: effect on some metabolic activities of the host. Lait $\mathbf{7 5}$, $435-445$.

Perez Chaia A \& Zarate G (2005) Dairy propionibacteria from milk or cheese diets remain viable and enhance propionic acid production in the mouse cecum. Lait 85, 85-98.

Perez Chaia A, Zarate G \& Oliver G (1999) The probiotic properties of propionibacteria. Lait 79, 175-185.

Rabot S, Szylit O, Nugon-Baudon L, Meslin JC, Vaissade P, Popot F \& Viso M (2000) Variations in digestive physiology of rats after short duration flights aboard the US space shuttle. Dig Dis Sci 45, 1687-1695.

Rigottier-Gois L, Le Bourhis AG, Gramet G, Rochet V \& Dore J (2003a) Fluorescent hybridisation combined with flow cytometry and hybridisation of total RNA to analyse the composition of microbial communities in human faeces using 16S rRNA probes. FEMS Microbiol Ecol 43, 237-245.

Rigottier-Gois L, Rochet V, Garrec N, Suau A \& Dore J (2003b) Enumeration of Bacteroides species in human faeces by fluorescent in situ hybridisation combined with flow cytometry using $16 \mathrm{~S}$ rRNA probes. Syst Appl Microbiol 26, 110-118.

Rumney CJ \& Rowland IR (1992) In vivo and in vitro models of the human colonic flora. Crit Rev Food Sci Nutr 31, 299-331.

Saarela M, Lahteenmaki L, Crittenden R, Salminen S \& MattilaSandholm T (2002) Gut bacteria and health foods - the European perspective. Int J Food Microbiol 78, 99-117.

Satomi K, Kurihara H, Isawa K, Mori H \& Kaneko T (1999) Effects of culture-powder of Propionibacterium freudenreichii ET-3 on fecal microflora of normal adults. Biosci Microfl 18, 27-30.

Scheppach W, Bartram HP \& Richter F (1995) Role of short-chain fatty acids in the prevention of colorectal cancer. Eur J Cancer 31A, 1077-1080.

Sghir A, Gramet G, Suau A, Rochet V, Pochart P \& Dore J (2000) Quantification of bacterial groups within human fecal flora by oligonucleotide probe hybridization. Appl Environ Microbiol 66, $2263-2266$.

Tannock GW (2001) Molecular assessment of intestinal microflora. Am J Clin Nutr 73, 410S-414S.

Wallner G, Amann R \& Beisker W (1993) Optimizing fluorescent in situ hybridization with rRNA-targeted oligonucleotide probes for flow cytometric identification of microorganisms. Cytometry 14, 136-143.

Warminska-Radyko I, Laniewska-Moroz L \& Babuchowski A (2002) Possibilities for stimulation of Bifidobacterium growth by propionibacteria. Lait 82, 113-121.

Wood HG (1981) Metabolic cycles in the fermentation of propionic acid bacteria. In Current Topics in Cellular Regulation, pp. 255-287 [RW Eastbrook and P Srera, editors]. New York: Academic Press.

Zarate G, Morata D, Chaia AP \& Gonzalez SN (2002) Adhesion of dairy propionibacteria to intestinal epithelial tissue in vitro and in vivo. J Food Prot 65, 534-539.

Zoetendal EG, Ben-Amor K, Akkermans AD, Abee T \& de Vos WM (2001) DNA isolation protocols affect the detection limit of PCR approaches of bacteria in samples from the human gastrointestinal tract. Syst Appl Microbiol 24, 405-410. 